УДК 517.954

\author{
H. P. Lopushanska, A. O. Lopushansky
}

\title{
UNIQUENESS OF SOLUTION FOR THE INVERSE PROBLEM OF FINDING TWO MINOR COEFFICIENTS IN A SEMILINEAR TIME FRACTIONAL TELEGRAPH EQUATION
}

\begin{abstract}
H. P. Lopushanska, A. O. Lopushansky. Uniqueness of solution for the inverse problem of finding two minor coefficients in a semilinear time fractional telegraph equation, Mat. Stud. 50 (2018), 189-197.

We find sufficient conditions of the uniqueness of a solution for the inverse problem of determining two continuous minor coefficients in a semilinear time fractional telegraph equation under two integral overdetermination conditions.
\end{abstract}

1. Introduction and preliminaries. The equations with fractional derivatives and inverse problems for them arise in many branches of science and engineering. The telegraph fractional equations in the theory of thermal stresses were considered, for example, in [1].

Some inverse problems to diffusion-wave equations with different unknown functions or parameters (source, order of partial derivative, elder or minor coefficient, boundary or initial data) were investigated, for example, in [2]-[13]. In particular, in papers $[2,4,5,7,8,9]$ the integral overdetermination conditions were used in the inverse source and coefficient problems for a time fractional diffusion equations.

In this paper, we find the conditions of the uniqueness of a classical solution $(u, r, b)$ of the inverse problem

$$
\begin{gathered}
D_{t}^{\alpha} u+r(t) D_{t}^{\beta} u=\Delta u+b(t) u+F_{0}\left(x, t, u, D_{t}^{\beta} u\right), \quad(x, t) \in \Omega \times(0, T], \\
u(x, t)=0, \quad(x, t) \in \partial \Omega \times[0, T], \\
u(x, 0)=F_{1}(x), \quad u_{t}(x, 0)=F_{2}(x), \quad x \in \bar{\Omega}, \\
\int_{\Omega} u(x, t) \varphi_{j}(x) d x=\Phi_{j}(t), t \in[0, T], \quad j=1,2
\end{gathered}
$$

where $\Omega$ is a bounded domain in $\mathbb{R}^{n}, n \in \mathbb{N}(n \geq 2)$ with the boundary $\partial \Omega$ of the class $C^{1+s}$, $s \in(0,1), \alpha \in(1,2), \beta \in(0,1), F_{0}, F_{1}, F_{2}, \Phi_{j}, \varphi_{j}(j=1,2)$ are given continuous functions.

2010 Mathematics Subject Classification: 35S10, 35S15.

Keywords: fractional derivative; inverse problem; integral overdetermination condition; Green's vectorfunction; integral equation.

doi:10.15330/ms.50.2.189-197

(C) H. P. Lopushanska, A. O. Lopushansky, 2018 
We note that inverse problems for semilinear parabolic and ultraparabolic equations with one unknown function were investigated, for example, in $[14,15]$ and for a semilinear time fractional telegraph equation in [7].

We shall use the method of Green's functions [16]-[20].

2. Main definitions and auxiliary results. Let $Q=\Omega \times(0, T], f * g$ be the convolution of functions $f$ and $g$,

$$
f_{\lambda}(t)=\frac{\theta(t) t^{\lambda-1}}{\Gamma(\lambda)} \text { for } \lambda>0 \text { and } f_{\lambda}(t)=f_{1+\lambda}^{\prime}(t) \text { for } \lambda \leq 0,
$$

where $\Gamma(t)$ is the Gamma-function, $\theta(t)$ is the Heaviside function. Note that

$$
f_{\lambda} * f_{\mu}=f_{\lambda+\mu}, \quad \lambda, \mu \in \mathbb{R}
$$

the Riemann-Liouville derivative $v_{t}^{(\alpha)}(x, t)$ of order $\alpha>0$ is defined by the formula

$$
v_{t}^{(\alpha)}(x, t)=f_{-\alpha}(t) * v(x, t)
$$

and the regularized fractional derivative is defined as follows

$$
\begin{gathered}
D_{t}^{\alpha} v(x, t)=\frac{1}{\Gamma(1-\alpha)}\left[\frac{\partial}{\partial t} \int_{0}^{t} \frac{v(x, \tau)}{(t-\tau)^{\alpha}} d \tau-\frac{v(x, 0)}{t^{\alpha}}\right]=v_{t}^{(\alpha)}(x, t)-f_{1-\alpha}(t) v(x, 0), \quad \alpha \in(0,1), \\
D_{t}^{\alpha} v(x, t)=\frac{1}{\Gamma(2-\alpha)} \int_{0}^{t} \frac{v_{\tau \tau}(x, \tau)}{(t-\tau)^{\alpha-1}} d \tau=v_{t}^{(\alpha)}(x, t)-f_{1-\alpha}(t) v(x, 0)-f_{2-\alpha}(t) v_{t}(x, 0), \\
\alpha \in(1,2) .
\end{gathered}
$$

Denote $D_{t}^{1} v=\frac{\partial v}{\partial t}$.

Let $C^{\gamma}(\Omega)\left(C^{\gamma}(Q)\right)$ be the space of bounded continuous functions on $\Omega$ ( $Q$, respectively) satisfying Hölder continuity condition (Hölder continuity condition with respect to space variables), $C^{2, \alpha}(Q)$ be the space of bounded continuous functions $v(x, t)$ on $Q$ together with the derivatives of the second order of the spatial variables and $D_{t}^{\alpha} v(x, t),(x, t) \in Q$, $C^{2, \alpha}(\bar{Q})=\left\{v \in C^{2, \alpha}(Q): v, v_{t} \in C(\bar{Q})\right\}$, for $\alpha \in(1,2)$.

Definition 1. A triple of functions

$$
(u, r, b) \in C^{2, \alpha}(\bar{Q}) \times C[0, T] \times C[0, T]
$$

satisfying equation (1) on $Q$ and conditions (2)-(4) is called a solution of problem (1)-(4).

The following necessary agreement conditions follow from the definition

$$
F_{1}=0 \text { on } \partial \Omega, \int_{\Omega} F_{1}(x) \varphi_{j}(x) d x=\Phi_{j}(0), \quad \int_{\Omega} F_{2}(x) \varphi_{j}(x) d x=\Phi_{j}^{\prime}(0), \quad j=1,2 .
$$

We denote $\left(L^{r e g} v\right)(x, t)=D_{t}^{\alpha} v(x, t)-\Delta v(x, t),(x, t) \in Q, v \in C^{2, \alpha}(Q)$.

Definition 2. A vector-function $\left(G_{0}(x, t, y, \tau), G_{1}(x, t, y), G_{2}(x, t, y)\right)$ is called a Green's vector-function of problem

$$
\left(L^{r e g} u\right)(x, t)=g_{0}(x, t), \quad(x, t) \in Q,
$$




$$
u=0 \text { on } \partial \Omega, \quad u(x, 0)=g_{1}(x), \quad u_{t}(x, 0)=g_{2}(x), \quad x \in \Omega,
$$

if under rather regular $g_{0}, g_{1}, g_{2}$, the function

$$
u(x, t)=\int_{0}^{t} d \tau \int_{\Omega} G_{0}(x, t, y, \tau) g_{0}(y, \tau) d y+\sum_{j=1}^{2} \int_{\Omega} G_{j}(x, t, y) g_{j}(y) d y, \quad(x, t) \in \bar{Q}
$$

is the classical solution (in $C^{2, \alpha}(\bar{Q})$ ) of problem $(5)-(6)$.

It follows from [20] that a Green's vector-function of problem (5)-(6) exists, for bounded $g_{0} \in C^{\gamma}(Q), g_{j} \in C^{\gamma}(\Omega), j=1,2$, the unique solution $u \in C^{2, \alpha}(\bar{Q})$ of problem (5)-(6) exists, defined by

$$
u(x, t)=\left(\mathcal{G}_{0} g_{0}\right)(x, t)+\left(\mathcal{G}_{1} g_{1}\right)(x, t)+\left(\mathcal{G}_{2} g_{2}\right)(x, t), \quad(x, t) \in \bar{Q}
$$

where

$$
\left(\mathcal{G}_{0} g_{0}\right)(x, t)=\int_{0}^{t} d \tau \int_{\Omega} G_{0}(x, t, y, \tau) g_{0}(y, \tau) d y, \quad\left(\mathcal{G}_{j} g_{j}\right)(x, t)=\int_{\Omega} G_{j}(x, t, y) g_{j}(y) d y, j=1,2 .
$$

3. Uniqueness of the solution. We pass to the inverse problem (1)-(4).

Theorem 1. Assume that $\alpha \in(1,2), \beta \in(0,1)$ and $F_{0} \in C^{1}\left(Q \times \mathbb{R}^{2}\right)$ is bounded, $\Phi_{j}, D^{\beta} \Phi_{j} \in$ $C[0, T], j=1,2$,

$$
d(t)=\Phi_{1}(t) D^{\beta} \Phi_{2}(t)-\Phi_{2}(t) D^{\beta} \Phi_{1}(t) \neq 0, \quad t \in[0, T],
$$

$\varphi_{j} \in C^{2}(\bar{\Omega}), j=1,2$,

$$
\varphi_{1}(x) \Delta \varphi_{2}(x)-\varphi_{2}(x) \Delta \varphi_{1}(x) \neq 0 \quad x \in \bar{\Omega} .
$$

Then the solution $(u, r, b) \in C^{2, \alpha}(\bar{Q}) \times C[0, T] \times C[0, T]$ of problem (1)-(4) is unique.

Proof. Take two solutions $\left(u_{1}, r_{1}, b_{1}\right),\left(u_{2}, r_{2}, b_{2}\right) \in C^{2, \alpha}(\bar{Q}) \times C[0, T] \times C[0, T]$ of problem (1)-(4) and substitute them into equation (1). For $u=u_{1}-u_{2}, r=r_{1}-r_{2}, b=b_{1}-b_{2}$ we obtain the equation

$$
\begin{aligned}
D_{t}^{\alpha} u= & \Delta u-r_{1}(t) D_{t}^{\beta} u-r(t) D_{t}^{\beta} u_{2}+b_{1}(t) u+b(t) u_{2} \\
& +F_{0}\left(x, t, u_{1}, D_{t}^{\beta} u_{1}\right)-F_{0}\left(x, t, u_{2}, D_{t}^{\beta} u_{2}\right) .
\end{aligned}
$$

By Hadamard's lemma

$$
F_{0}\left(x, t, u_{1}, D_{t}^{\beta} u_{1}\right)-F_{0}\left(x, t, u_{2}, D_{t}^{\beta} u_{2}\right)=F_{01}(x, t) u+F_{02}(x, t) D_{t}^{\beta} u
$$

with some known functions $F_{0 j}, j=1,2$, which are continuous and bounded on $Q$. Then the previous equation gets the form

$$
\begin{gathered}
D_{t}^{\alpha} u+\left(r_{1}(t)-F_{02}(x, t)\right) D_{t}^{\beta} u \\
=\Delta u+\left(F_{01}(x, t)+b_{1}(t)\right) u-r(t) D_{t}^{\beta} u_{2}+b(t) u_{2}, \quad(x, t) \in Q .
\end{gathered}
$$


It follows from the conditions (2)-(4) that

$$
\begin{gathered}
u=0 \text { on } \partial \Omega, \quad u(x, 0)=0, \quad u_{t}(x, 0)=0, \quad x \in \bar{\Omega}, \\
\int_{\Omega} u(x, t) \varphi_{j}(x) d x=0, \quad t \in[0, T], \quad j=1,2 .
\end{gathered}
$$

Let $G_{0}^{*}(x, t, y, \tau)$ be the main Green's function [17, 18, 20] of problem (2)-(3) to the equation

$$
D_{t}^{\alpha} u+\left(r_{1}(t)-F_{02}(x, t)\right) D_{t}^{\beta} u=\Delta u+\left(F_{01}(x, t)+b_{1}(t)\right) u .
$$

Then the function $u(x, t)$ gets the form

$$
u(x, t)=\int_{0}^{t} d \tau \int_{\Omega} G_{0}^{*}(x, t, y, \tau)\left[u_{2}(y, \tau) b(\tau)-D_{\tau}^{\beta} u_{2}(y, \tau) r(\tau)\right] d y, \quad(x, t) \in \bar{\Omega} .
$$

It follows from the equation and overdetermination conditions that

$$
\begin{gathered}
r(t) D^{\beta} \Phi_{j}(t)-b(t) \Phi_{j}(t)=\int_{\Omega}\left[u(x, t) \Delta \varphi_{j}(x)\right. \\
\left.+\left(F_{01}(x, t) u(x, t)+F_{02}(x, t) D_{t}^{\beta} u(x, t)\right) \varphi_{j}(x)\right] d x, \quad t \in[0, T], \quad j=1,2 .
\end{gathered}
$$

From here and assumption (9) we find

$$
\begin{gathered}
r(t)=\frac{\Phi_{1}(t)}{d(t)} \int_{\Omega}\left[u(z, t) \Delta \varphi_{2}(z)+\left(F_{01}(z, t) u(z, t)+F_{02}(z, t) D_{t}^{\beta} u(z, t)\right) \varphi_{2}(z)\right] d z \\
-\frac{\Phi_{2}(t)}{d(t)} \int_{\Omega}\left[u(z, t) \Delta \varphi_{1}(z)+\left(F_{01}(z, t) u(z, t)+F_{02}(z, t) D_{t}^{\beta} u(z, t)\right) \varphi_{1}(z)\right] d z \\
b(t)=\frac{D^{\beta} \Phi_{1}(t)}{d(t)} \int_{\Omega}\left[u(z, t) \Delta \varphi_{2}(z)+\left(F_{01}(z, t) u(z, t)+F_{02}(z, t) D_{t}^{\beta} u(z, t)\right) \varphi_{2}(z)\right] d z \\
-\frac{D^{\beta} \Phi_{2}(t)}{d(t)} \int_{\Omega}\left[u(z, t) \Delta \varphi_{1}(z)+\left(F_{01}(z, t) u(z, t)+F_{02}(z, t) D_{t}^{\beta} u(z, t)\right) \varphi_{1}(z)\right] d z, \quad t \in[0, T] .
\end{gathered}
$$

Substituting those expressions for $r(t)$ and $b(t)$ (by $u, D_{t}^{\beta} u$ ) in (11) we obtain the equation

$$
\begin{aligned}
& u(x, t)= \int_{0}^{t} \frac{1}{d(\tau)}\left\{-K_{1}^{*}(x, t, \tau) \int_{\Omega}\left[\left(\Phi_{1}(\tau)\left(F_{01}(z, \tau) \varphi_{2}(z)+\Delta \varphi_{2}(z)\right)\right.\right.\right. \\
&\left.\quad-\Phi_{2}(\tau)\left(F_{01}(z, \tau) \varphi_{1}(z)+\Delta \varphi_{1}(z)\right)\right) u(z, \tau) \\
&+\left.\left(\Phi_{1}(\tau) \varphi_{2}(z)-\Phi_{2}(\tau) \varphi_{1}(z)\right) F_{02}(z, \tau) D_{\tau}^{\beta} u(z, \tau)\right] d z \\
&+ K_{2}^{*}(x, t, \tau) \int_{\Omega}\left[\left(D^{\beta} \Phi_{1}(\tau)\left(F_{01}(z, \tau) \varphi_{2}(z)+\Delta \varphi_{2}(z)\right)\right.\right. \\
&\left.\quad-D^{\beta} \Phi_{2}(\tau)\left(F_{01}(z, \tau) \varphi_{1}(z)+\Delta \varphi_{1}(z)\right)\right) u(z, \tau) \\
&\left.\left.+\left(D^{\beta} \Phi_{1}(\tau) \varphi_{2}(z)-D^{\beta} \Phi_{2}(\tau) \varphi_{1}(z)\right) F_{02}(z, \tau) D_{\tau}^{\beta} u(z, \tau)\right] d z\right\} d \tau, \quad(x, t) \in \bar{Q}
\end{aligned}
$$


where

$$
\begin{gathered}
K_{1}^{*}(x, t, \tau)=\int_{\Omega} G_{0}^{*}(x, t, y, \tau) D_{\tau}^{\beta} u_{2}(y, \tau) d y \\
K_{2}^{*}(x, t, \tau)=\int_{\Omega} G_{0}^{*}(x, t, y, \tau) u_{2}(y, \tau) d y, \quad x \in \Omega, 0 \leq \tau<t \leq T
\end{gathered}
$$

are the known functions. According to $[17,19]$ the function $G_{0}^{*}(x, t, y, \tau)$ has the same kind of estimates as $G_{0}(x, t, y, \tau)$ has. Then it follows from [8, Lemma 1], for example, that $K_{j}^{*}(x, t, \tau)$ are continuous functions in $x \in \Omega$ and

$$
\left|K_{j}^{*}(x, t, \tau)\right| \leq C_{j}(t-\tau)^{\alpha-1}, \quad x \in \Omega, 0 \leq \tau<t \leq T, \quad j=1,2 .
$$

Hereinafter $C_{j}, C_{j}^{*}, \widehat{C}_{j}(j=1,2)$ are positive constants.

In the case of linear equation (1) we have $F_{01}(z, \tau)=F_{02}(z, \tau)=0,(z, \tau) \in Q$ and equation (14) gets a form

$$
\begin{gathered}
u(x, t)=\int_{0}^{t} \frac{d \tau}{d(\tau)} \int_{\Omega}\left[-K_{1}^{*}(x, t, \tau)\left(\Phi_{1}(\tau) \Delta \varphi_{2}(z)-\Phi_{2}(\tau) \Delta \varphi_{1}(z)\right)\right. \\
\left.+K_{2}^{*}(x, t, \tau)\left(D^{\beta} \Phi_{1}(\tau) \Delta \varphi_{2}(z)-D^{\beta} \Phi_{2}(\tau) \Delta \varphi_{1}(z)\right)\right] u(z, \tau) d z, \quad(x, t) \in \bar{Q} .
\end{gathered}
$$

It is the homogeneous linear Volterra integral equation of the second type with the continuous kernel which has the unique solution $u(x, t)=0,(x, t) \in \bar{Q}$. Note that

$$
\begin{gathered}
D_{t}^{\beta} u(x, t)=0 \Longleftrightarrow f_{-\beta}(t) * u(x, t)=0 \Longleftrightarrow f_{\beta}(t) * f_{-\beta}(t) * u(x, t)=0 \\
\Longleftrightarrow u(x, t)=0, \quad(x, t) \in Q,
\end{gathered}
$$

for the function $u(x, t)$ satisfying zero initial conditions. Then we have

$$
u(x, t)=D_{t}^{\beta} u(x, t)=0, \quad(x, t) \in \bar{Q}
$$

and from (13) we obtain $r(t)=b(t)=0, t \in[0, T]$.

Now assume that

$$
\left|F_{01}(x, t)\right|+\left|F_{02}(x, t)\right| \neq 0, \quad(x, t) \in Q .
$$

Then assumption (10) implies that at least one of the following assumptions

$$
\left|F_{01}(x, t) \varphi_{j}(x)+\Delta \varphi_{j}(x)\right|+\left|F_{02}(x, t)\right| \neq 0, \quad(x, t) \in Q, \quad j=1,2 .
$$

holds.

If $F_{02}(z, \tau)=0,(z, \tau) \in Q$ then by the unique solvability of the linear homogeneous second type Volterra integral equation

$$
\begin{gathered}
u(x, t)=\int_{0}^{t} \frac{d \tau}{d(\tau)} \int_{\Omega}\left[-K_{1}^{*}(x, t, \tau)\left(\Phi_{1}(\tau)\left(F_{01}(z, \tau) \varphi_{2}(z)+\Delta \varphi_{2}(z)\right)\right.\right. \\
\left.-\Phi_{2}(\tau)\left(F_{01}(z, \tau) \varphi_{1}(z)+\Delta \varphi_{1}(z)\right)\right)
\end{gathered}
$$




$$
\begin{gathered}
+K_{2}^{*}(x, t, \tau)\left(D^{\beta} \Phi_{1}(\tau)\left(F_{01}(z, \tau) \varphi_{2}(z)+\Delta \varphi_{2}(z)\right)\right. \\
\left.\left.-D^{\beta} \Phi_{2}(\tau)\left(F_{01}(z, \tau) \varphi_{1}(z)+\Delta \varphi_{1}(z)\right)\right)\right] u(z, \tau) d z, \quad(x, t) \in \bar{Q}
\end{gathered}
$$

with the integrable kernel we obtain $u(x, t)=0,(x, t) \in Q$. As above, we find $r(t)=b(t)=0$, $t \in[0, T]$.

In general case, denote

$$
\mu_{j}(x, t)=F_{01}(x, t) \varphi_{j}(x)+\Delta \varphi_{j}(x), \quad j=1,2 .
$$

Then equation (14) gets a form

$$
\begin{aligned}
& u(x, t)=\int_{0}^{t} \frac{d \tau}{d(\tau)} \int_{\Omega}\left\{\left[\left(-K_{1}^{*}(x, t, \tau) \Phi_{1}(\tau)+K_{2}^{*}(x, t, \tau) D^{\beta} \Phi_{1}(\tau)\right) \mu_{2}(z, \tau)\right.\right. \\
&\left.-\left(-K_{1}^{*}(x, t, \tau) \Phi_{2}(\tau)+K_{2}^{*}(x, t, \tau) D^{\beta} \Phi_{2}(\tau)\right) \mu_{1}(z, \tau)\right] u(z, \tau) \\
&+F_{02}(z, \tau)\left[\left(-K_{1}^{*}(x, t, \tau) \Phi_{1}(\tau)+K_{2}^{*}(x, t, \tau) D^{\beta} \Phi_{1}(\tau)\right) \varphi_{2}(z)\right. \\
&\left.\left.-\left(-K_{1}^{*}(x, t, \tau) \Phi_{2}(\tau)+K_{2}^{*}(x, t, \tau) D^{\beta} \Phi_{2}(\tau)\right) \varphi_{1}(z)\right] D_{\tau}^{\beta} u(z, \tau)\right\} d z, \quad(x, t) \in \bar{Q} .
\end{aligned}
$$

Denoting $a_{j}(x, t, \tau)=-K_{1}^{*}(x, t, \tau) \Phi_{j}(\tau)+K_{2}^{*}(x, t, \tau) D^{\beta} \Phi_{j}(\tau), w_{j}(x, t)=\mu_{j}(x, t) u(x, t)+$ $F_{02}(x, t) \varphi_{j}(x) D_{t}^{\beta} u(x, t), j=1,2$, we get

$$
u(x, t)=\int_{0}^{t} \frac{1}{d(\tau)}\left\{a_{1}(x, t, \tau) \int_{\Omega} w_{2}(z, \tau) d z-a_{2}(x, t, \tau) \int_{\Omega} w_{1}(z, \tau) d z\right\} d \tau, \quad(x, t) \in \bar{Q} .
$$

Note that

$$
\begin{gathered}
D_{t}^{\beta} \int_{0}^{t} a(t, \tau) d \tau=f_{-\beta}(t) * \int_{0}^{t} a(t, \tau) d \tau=f_{1-\beta}(t) * \frac{\partial}{\partial t} \int_{0}^{t} a(t, \tau) d \tau \\
=\frac{1}{\Gamma(1-\beta)} \int_{0}^{t}(t-s)^{-\beta}\left(\frac{\partial}{\partial s} \int_{0}^{s} a(s, \tau) d \tau\right) d s=\int_{0}^{t} \frac{d \tau}{\Gamma(1-\beta)} \int_{\tau}^{t} \frac{\partial}{\partial s} a(s, \tau)(t-s)^{-\beta} d s
\end{gathered}
$$

if $a(t, t)=0, t \in[0, T]$ and the right-hand side of this expression exists.

It follows from the definitions of $a_{j}(x, t, \tau),(15)$ and [8, Lemma 1] (where the case of a linear equation was studied) that

$$
\begin{aligned}
a_{j}(x, s, s) & =0, \quad\left|a_{j}(x, s, \tau)\right| \leq C_{j}^{*}(s-\tau)^{\alpha-1}, \\
\left|\int_{\tau}^{t} \frac{\partial}{\partial s} a_{j}(x, s, \tau)(t-s)^{-\beta} d s\right| & \leq \widehat{C}_{j}(t-\tau)^{\alpha-\beta-1}, \quad x \in \bar{\Omega}, \quad 0 \leq \tau<s \leq T, \quad j=1,2 .
\end{aligned}
$$

From (20) we obtain

$$
w_{1}(x, t)=\int_{0}^{t} \frac{1}{d(\tau)}\left\{\mu_{1}(x, t)\left[a_{1}(x, t, \tau) \int_{\Omega} w_{2}(z, \tau) d z-a_{2}(x, t, \tau) \int_{\Omega} w_{1}(z, \tau) d z\right]\right.
$$




$$
\begin{gathered}
+\frac{F_{02}(x, t) \varphi_{1}(x)}{\Gamma(1-\beta)}\left[\int_{\tau}^{t} \frac{\partial}{\partial s} a_{1}(x, s, \tau)(t-s)^{-\beta} d s \int_{\Omega} w_{2}(z, \tau) d z\right. \\
\left.\left.\quad-\int_{\tau}^{t} \frac{\partial}{\partial s} a_{2}(x, s, \tau)(t-s)^{-\beta} d s \int_{\Omega} w_{1}(z, \tau) d z\right]\right\} d \tau \\
w_{2}(x, t)=\int_{0}^{t} \frac{1}{d(\tau)}\left\{\mu_{2}(x, t)\left[a_{1}(x, t, \tau) \int_{\Omega} w_{2}(z, \tau) d z-a_{2}(x, t, \tau) \int_{\Omega} w_{1}(z, \tau) d z\right]\right. \\
+\frac{F_{02}(x, t) \varphi_{2}(x)}{\Gamma(1-\beta)}\left[\int_{\tau}^{t} \frac{\partial}{\partial s} a_{1}(x, s, \tau)(t-s)^{-\beta} d s \int_{\Omega} w_{2}(z, \tau) d z\right. \\
\left.\left.-\int_{\tau}^{t} \frac{\partial}{\partial s} a_{2}(x, s, \tau)(t-s)^{-\beta} d s \int_{\Omega} w_{1}(z, \tau) d z\right]\right\} d \tau,
\end{gathered}
$$

that is

$$
\left(\begin{array}{l}
w_{1}(x, t) \\
w_{2}(x, t)
\end{array}\right)=\int_{0}^{t} \frac{H(x, t, \tau) d \tau}{d(\tau)} \int_{\Omega}\left(\begin{array}{l}
w_{1}(z, \tau) \\
w_{2}(z, \tau)
\end{array}\right) d z, \quad(x, t) \in \bar{Q}
$$

where

$$
\begin{gathered}
H(x, t, \tau)=\left(\begin{array}{cc}
H_{11}(x, t, \tau) & H_{12}(x, t, \tau) \\
H_{21}(x, t, \tau) & H_{22}(x, t, \tau)
\end{array}\right) \\
H_{11}(x, t, \tau)=-\mu_{1}(x, t) a_{2}(x, t, \tau)-\frac{F_{02}(x, t) \varphi_{1}(x)}{\Gamma(1-\beta)} \int_{\tau}^{t} \frac{\partial}{\partial s} a_{2}(x, s, \tau)(t-s)^{-\beta} d s \\
H_{12}(x, t, \tau)=\mu_{1}(x, t) a_{1}(x, t, \tau)+\frac{F_{02}(x, t) \varphi_{1}(x)}{\Gamma(1-\beta)} \int_{\tau}^{t} \frac{\partial}{\partial s} a_{1}(x, s, \tau)(t-s)^{-\beta} d s \\
H_{21}(x, t, \tau)=-\mu_{2}(x, t) a_{2}(x, t, \tau)-\frac{F_{02}(x, t) \varphi_{2}(x)}{\Gamma(1-\beta)} \int_{\tau}^{t} \frac{\partial}{\partial s} a_{2}(x, s, \tau)(t-s)^{-\beta} d s \\
H_{22}(x, t, \tau)=\mu_{2}(x, t) a_{1}(x, t, \tau)+\frac{F_{02}(x, t) \varphi_{2}(x)}{\Gamma(1-\beta)} \int_{\tau}^{t} \frac{\partial}{\partial s} a_{1}(x, s, \tau)(t-s)^{-\beta} d s
\end{gathered}
$$

Taking bounds (21) into account we see that the matrix function $H(x, t, \tau)$ is continuous in $x \in \bar{\Omega}$ and integrable in $t$ and $\tau$. Then the linear homogeneous second type Volterra system of integral equations (22) has (see, for example, [21, p. 60]) the unique continuous solution $w_{1}(x, t)=w_{2}(x, t)=0,(x, t) \in \bar{Q}$. By the definition of $w_{j}(j=1,2)$ we get the linear homogeneous algebraic system of equations

$$
\begin{aligned}
& \mu_{1}(x, t) u(x, t)+F_{02}(x, t) \varphi_{1}(x) D_{t}^{\beta} u(x, t)=0, \\
& \mu_{2}(x, t) u(x, t)+F_{02}(x, t) \varphi_{2}(x) D_{t}^{\beta} u(x, t)=0
\end{aligned}
$$


for finding $u(x, t)$ and $D_{t}^{\beta} u(x, t),(x, t) \in \bar{Q}$. Its determinant is equal to

$$
F_{02}(x, t)\left[\mu_{1}(x, t) \varphi_{2}(x)-\mu_{2}(x, t) \varphi_{1}(x)\right]=F_{02}(x, t)\left[\varphi_{2}(x) \Delta \varphi_{1}(x)-\varphi_{1}(x) \Delta \varphi_{2}(x)\right] .
$$

Then, taking the assumptions (10) and (18) into account we find solution (16) of system (23). In fact, this is obvious when $F_{02}(x, t) \neq 0,(x, t) \in Q$. In the case $F_{02}(x, t)=0$ for some $(x, t) \in Q$, by $(18)$ we have

$$
F_{01}(x, t) \varphi_{1}(x)+\Delta \varphi_{1}(x) \neq 0 \text { or } F_{01}(x, t) \varphi_{2}(x)+\Delta \varphi_{2}(x) \neq 0,
$$

that is $\mu_{1}(x, t) \neq 0$ or $\mu_{2}(x, t) \neq 0$ for such $(x, t) \in Q$, respectively, and system (23) implies $u(x, t)=0,(x, t) \in Q$. Then, as in the previous case, we get (16), and (13) implies $r(t)=$ $b(t)=0, t \in[0, T]$.

Remark. In the particular case $F_{0}=F_{0}(x, t, u)$ the claim of the Theorem remains correct without condition (10). This case was studied in the proof $\left(F_{02}(x, t)=0,(x, t) \in Q\right)$.

\section{REFERENCES}

1. Y. Povstenko, Theories of thermal stresses based on space-time fractional telegraph equations, Comput. Math. Appl., 64 (2012), 3321-3328.

2. T.S. Aleroev, M. Kirane, S.A. Malik, Determination of a source term for a time fractional diffusion equation with an integral type overdetermination condition, Electron. J. Differ. Equ., 2013 (2013), 1-16.

3. Y. Hatano, J. Nakagawa, Sh. Wang, M. Yamamoto, Determination of order in fractional diffusion equation, J. Math-for-Ind., 5A (2013), 51-57.

4. M.I. Ismailov, Inverse source problem for a time-fractional diffusion equation with nonlocal boundary conditions, Appl. Math. Model., 40 (2016), №7/8, 4891-4899.

5. J. Janno, K. Kasemets, Uniqueness for an inverse problem foe a semilinear time-fractional diffusion equation, // Inverse Probl. Imaging, 11 (2017), №1, 125-149. doi: 10.3934/ipi.2017007

6. B. Jin, W. Rundell, A turorial on inverse problems for anomalous diffusion processes, Inverse Probl., 31 (2015), №3. doi:10.1088/0266-5611/31/3/035003.

7. H. Lopushanska, V. Rapita, Inverse coefficient problem for semi-linear fractional telegraph equation, Electron. J. Differ. Equ., 2015 (2015), №153, 1-13.

8. H. Lopushanska, V. Shumska, The inverse problem of finding minor coefficients in the telegraph equation with fractional derivatives in time, In: Nonclassical problems of the theory of differential equations: a collection of scientific works devoted to the 80th anniversary of B.Y. Ptashnyk. Under the Ed. Kushnir R.M., Pelykh V.O., Lviv: Ya.S. Pidstryhach IAPMM of NAS of Ukraine, 2017. 161-172. (in Ukrainian)

9. H. Lopushanska, A problem with an integral boundary condition for a time fractional diffusion equation and an inverse problem, Fract. Differ. Calc., 6 (2016), №1, 133-145. doi:10.7153/fdc-06-09

10. A. Lopushanskyj, Solvability of inverse boundary value problem for equation with fractional derivative, Visn. Lviv Univ. Ser. Mech. Math., 79 (2014), 97-110. (in Ukrainian)

11. W. Rundell, X. Xu, L. Zuo, The determination of an unknown boundary condition in fractional diffusion equation, Appl. Anal., 1 (2012), 1-16.

12. K. Sakamoto, M. Yamamoto, Initial value/boundary-value problems for fractional diffusion-wave equations and applications to some inverse problems, J. Math. Anal. Appl., 382 (2011), №1, 426-447.

13. Y. Zhang, X. Xu, Inverse source problem for a fractional diffusion equation, Inv. probl., 27 (2011), 1-12.

14. M. Ivanchov, Inverse problem for semilinear parabolic equation, Mat. Stud., 29 (2008), №2, $181-191$.

15. N.P. Protsakh, Asymptotic behavior of solution of the inverse problem for weakly nonlinear ultraparabolic equation, Carpathian Math. Publ., 5 (2013), №2, 326-335. doi: 10.15330/cmp.5.2.326-335 (in Ukrainian) 
16. V.V. Anh, N.N. Leonenko, Spectral analysis of fractional kinetic equations with random datas, J. Statist. Phys., 104 (2001), №5/6, 1349-1387.

17. S.D. Eidelman, S.D. Ivasyshen, A.N. Kochubei, Analytic methods in the theory of differential and pseudodifferential equations of parabolic type, Basel-Boston-Berlin, Birkhauser Verlag, 2004.

18. A. Friedman, Partial differential equations of parabolic type, Englewood Cliffs, N. J., Prentice-Hall, 1964.

19. M.I. Matijchuk, The connection between fundamental solutions of parabolic equations and fractional equations, Bukovyn Math. J., 4 (2016), №3-4, 101-114. (in Ukrainian)

20. A.A. Voroshylov, A.A. Kilbas, Conditions of the existence of classical solution of the Cauchy problem for diffusion-wave equation with Caputo partial derivative, Dokl. Ak. Nauk., 414 (2007), №4, 1-4.

21. P.P. Zabreiko, A.I. Koshelev, M.A. Krasnoselsky, S.G. Mikhlin, L.S. Rakovshchik, V.Ya. Stetsenko, Integral equations, Nauka, Moskow, 1968. (in Russian)

Ivan Franko National University of Lviv, Lviv, Ukraine

lhp@ukr.net

Faculty of Mathematical and Natural Sciences, Rzeszów University

Rzeszów, Poland

alopushanskyj@gmail.com 